\title{
A COMPACTIFICATION OF LOCALLY COMPACT SPACES
}

\author{
F. W. LOZIER ${ }^{1}$
}

\begin{abstract}
Every locally compact space $X$ has its topology determined by its 1-1 compact images and hence has a compactification $\xi X$ obtained as the closure of the natural embedding of $X$ in the product of those images, just as the Stone-Cech compactification $\beta X$ can be obtained by embedding $X$ in a product of intervals. The obvious question is whether $\xi X=\beta X$. In this paper we prove that $\xi X=\beta X$ if $X$ either is 0 -dimensional or contains an arc, and give an example in which $\xi X \neq \beta X$.
\end{abstract}

Preliminaries. All maps are continuous, and all compact and locally compact spaces are Hausdorff. For any space $X$, let $\mathscr{K}(X)$ denote the set of all 1-1 maps of $X$ onto a compact space, let $Y=\prod\{f(X) \mid f \in \mathscr{K}(X)\}$ and let $e: X \rightarrow Y$ be the evaluation map; if $e$ is a homeomorphism then $\operatorname{cl}_{Y} e(X)$ is a compactification of $X$ which we denote by $\xi X$. If $X$ is locally compact then $e$ is necessarily a homeomorphism because for any closed $F \subseteq X$ there is an $f \in \mathscr{K}(X)$ such that $f(F)$ is closed in $f(X)$ : choose $x \in F$, let $X^{\prime}$ be the set $X$ with the topology consisting of all open $U \subseteq X$ such that either $x \notin U$ or $X-U$ is compact, and let $f: X \rightarrow X^{\prime}$ be the natural map. Note that, by a standard argument, $\xi X$ is the smallest compactification of $X$ to which every $f \in \mathscr{K}(X)$ can be extended.

Proposition. Suppose that for any two disjoint zero-sets $Z_{1}$ and $Z_{2}$ of a locally compact space $X$ there is a map from $X$ into a compact subspace $Y$ of $X$ such that $f\left(Z_{1}\right)$ and $f\left(Z_{2}\right)$ have disjoint closures. Then $\xi X=\beta X$.

Proof. Let $\tilde{e}: \beta X \rightarrow \xi X$ be the Stone extension of the embedding $e: X \rightarrow \xi X$. Since $e$ is a homeomorphism it follows that $\tilde{e}(\beta X-X)=$ $\xi X-e(X)$; hence we need only show that $\tilde{e}(p) \neq \tilde{e}(q)$ for any two distinct $p, q \in \beta X-X$. Let $A_{p}$ and $A_{q}$ be the free $z$-ultrafilters on $X$ converging to $p$ and $q$, respectively, choose disjoint $Z_{1} \in A_{p}$ and $Z_{2} \in A_{q}$, and let $f$ and $Y$ be as hypothesized. Then let $\tilde{f}: \beta X \rightarrow Y$ be the Stone extension of $f$, let $Y^{\prime}=$ $\tilde{f}(\beta X-X)$, let $X^{\prime}=(\beta X-X) \cup Y^{\prime}$, and define $g: X^{\prime} \rightarrow Y^{\prime}$ by requiring that

Received by the editors February 4, 1971.

AMS 1969 subject classification. Primary 5453.

Key words and phrases. Compactification, Stone-Čech compactification.

1 This paper is drawn from the author's doctoral dissertation, directed by Professor S. W. Willard at Case Western Reserve University, 1968.

(c) American Mathematical Society 1972 
$g(x)$ be $\tilde{f}(x)$ or $x$ according as $x \in \beta X-X$ or $x \in Y^{\prime}$. Since $\beta X-X$ and $Y^{\prime}$ are disjoint closed subsets of $\beta X, g$ is continuous. Therefore, since $X^{\prime}$ is compact and $Y^{\prime}$ is Hausdorff, $\mathscr{D}^{\prime}=\left\{g^{-1}(y) \mid y \in Y^{\prime}\right\}$ is a closed, upper semicontinuous decomposition of $X^{\prime}$, i.e., $\bigcup\left\{D \in \mathscr{D}^{\prime} \mid D \cap F \neq \varnothing\right\}$ is closed for every closed $F \subseteq X^{\prime}$. As a consequence, if $\mathscr{D}=\mathscr{D}^{\prime} \cup\left\{\{x\} \mid x \in X-Y^{\prime}\right\}$ then, for any closed $F \subseteq \beta X$,

$$
\bigcup\{D \in \mathscr{D} \mid D \cap F \neq \varnothing\}=F \cup\left(\bigcup\left\{D \in \mathscr{D}^{\prime} \mid D \cap\left(F \cap X^{\prime}\right) \neq \varnothing\right\}\right)
$$

is closed in $\beta X$, so that $\mathscr{D}$ is a closed, upper semicontinuous decomposition of $\beta X$. Thus, if $h$ is the projection of $\beta X$ onto the quotient space determined by $\mathscr{D}$, then $h(\beta X)$ is Hausdorff and hence compact. Let $k=h \mid X$. Then $k \in \mathscr{K}(X)$ so that there is a map $\hat{k}: \xi X \rightarrow k(X)$ such that $\hat{k}_{\circ} e=k$. Now $Z_{1} \in A_{p}$ so that $p \in \mathrm{cl}_{\beta X} Z_{1}$ and hence $g(p)=\tilde{f}(p) \in \mathrm{cl} f\left(Z_{1}\right)$. Similarly, $g(q) \in \mathrm{cl} f\left(Z_{2}\right)$. Therefore, since $\mathrm{cl} f\left(Z_{1}\right)$ and $\mathrm{cl} f\left(Z_{2}\right)$ are disjoint, it follows that $g(p) \neq g(q)$ and hence, by the definition of $h$, that $h(p) \neq h(q)$. But $\hat{k} \circ \tilde{e}$ and $h$ agree on the dense subset $X$ of $\beta X$ so that $\hat{k} \circ \tilde{e}=h$. Hence $\tilde{e}(p) \neq \tilde{e}(q)$, as required.

COROLlary 1. If a locally compact space is 0 -dimensional in the sense of [3], then $\xi X=\beta X$.

Proof. Any two disjoint zero-sets $Z_{1}$ and $Z_{2}$ of $X$ are contained in disjoint open sets $U_{1}$ and $U_{2}$ whose union is $X$. Choose $x_{i} \in U_{i}$, let $Y=$ $\left\{x_{1}, x_{2}\right\}$, and define $f: X \rightarrow Y$ by requiring $f(x)=x_{i}$ if $x \in U_{i}$.

COROLlary 2. If a locally compact space $X$ contains an arc, then $\xi X=\beta X$.

Proof. By assumption, there is a map $g:[0,1] \rightarrow X$ such that $g(0) \neq g(1)$. For any two disjoint zero-sets $Z_{1}$ and $Z_{2}$ of $X$, there is a map $h: X \rightarrow[0,1]$ such that $h\left(Z_{1}\right)=0$ and $h\left(Z_{2}\right)=1$. Let $f=g \circ h$.

EXAMPLE. According to Cook [1] there is a nontrivial, compact, connected space which admits no map into itself other than the identity map and the constant maps. Let $C$ be such a space and let $\alpha$ be the first ordinal with card $\alpha>\operatorname{card} C$. Note that necessarily card $\alpha$ is uncountable. Now let $x_{1}$ and $x_{2}$ be distinct points of $C$, let $p$ be a point not in $C$, and set $H=C \times[0, \alpha] \times[0, \alpha]$ and $K=\{p\} \times[0, \alpha] \times[0, \alpha]$. Then

$$
A=\left(\left\{x_{1}\right\} \times\{\alpha\} \times[0, \alpha]\right) \cup\left(\left\{x_{2}\right\} \times[0, \alpha] \times\{\alpha\}\right)
$$

is closed in $H$ and the map $\theta: A \rightarrow K$ defined by requiring $f(\langle x, \beta, \gamma\rangle)=$ $\langle p, \beta, \gamma\rangle$ is continuous, so that [2, VI.6.1 and VII.3.4] the space $Y=H \cup_{\theta} K$ obtained by "attaching $H$ to $K$ by $\theta$ " is compact. Let $Y^{\prime}$ be the free union of $H$ and $K$ and let $\psi: Y^{\prime} \rightarrow Y$ be the quotient map. Let

$$
X^{\prime}=Y^{\prime}-((C \cup\{p\}) \times\{\alpha\} \times\{\alpha\})
$$


and let $X=\psi\left(X^{\prime}\right)$. Then $Y-X=\psi\left(Y^{\prime}-X^{\prime}\right)$ is closed in $Y$, and hence $X$ is locally compact. Moreover, $X$ is dense in $Y$ so that, in order to show that $\beta X=Y$, it suffices to show that any $f \in C^{*}(X)$ can be extended to $f \in C^{*}(Y)$. But $g=f \circ\left(\psi \mid X^{\prime}\right) \in C^{*}\left(X^{\prime}\right)$ so that, by standard techniques $[3,8 \mathrm{~L}$ and $9 \mathrm{~K}]$, one can show that there is a $\beta<\alpha$ such that $g$ is constant on $X^{\prime} \cap(\{x\} \times[\beta, \alpha] \times[\beta, \alpha])$ for all $x \in C \cup\{p\}$. Hence $g$ can be extended to $\tilde{g} \in C^{*}\left(Y^{\prime}\right)$ by setting $\tilde{g}(\langle x, \alpha, \alpha\rangle)=g(\langle x, \beta, \beta\rangle)$. Moreover, $\tilde{g}\left(\left\langle x_{1}, \alpha, \alpha\right\rangle\right)=$ $g\left(\left\langle x_{1}, \beta, \beta\right\rangle\right)=g\left(\left\langle x_{1}, \alpha, \beta\right\rangle\right)=g(\langle p, \alpha, \beta\rangle)=g(\langle p, \beta, \beta\rangle)=\tilde{g}(\langle p, \alpha, \alpha\rangle) \quad$ and, similarly, $\tilde{g}\left(\left\langle x_{2}, \alpha, \alpha\right\rangle\right)=\tilde{g}(\langle p, \alpha, \alpha\rangle)$ so that there is an $\tilde{f} \in C^{*}(Y)$ such that $\tilde{f} \circ \psi=\tilde{g}$, the continuity of $\tilde{f}$ following from the fact that $\psi$ is a quotient map. Clearly $\tilde{f}$ is the desired extension of $f$ so that $\beta X=Y$ as asserted.

Now consider any $f \in \mathscr{K}(X)$, let $\tilde{f}: Y \rightarrow f(X)$ be the Stone extension of $f$, and let $D=f^{-1}(\tilde{f}(Y-X))$. Then $D$ is a closed subset of $X$ with card $D \leqq$ card $(Y-X)=$ card $C<$ card $\alpha$, so that $\psi^{-1}(D)$ is a closed subset of $X^{\prime}$ of cardinality less than card $\alpha$ and hence, by a standard argument, compact. Thus $D$ is compact and hence a continuous image of $Y-X$ under the $\operatorname{map} f^{-1} \circ \tilde{f}$. But $Y-X$ is just $C$ with the points $x_{1}$ and $x_{2}$ identified, so that $D$ is a continuous image of $C$ under a map which is not 1-1. Therefore, since every component of $X$ is either a singleton or a homeomorph of $C$, it follows that $D$ consists of a single point. Thus $\tilde{f}(Y-X)$ is a singleton so that $f$ can be extended to the one-point compactification of $X$ obtained by identifying $Y-X$ into a point. Hence $\xi X$ is just the one-point compactification of $X$; in particular, $\xi X \neq \beta X$.

REMARK. The obvious open problem is to find an internal characterization of the locally compact spaces $X$ for which $\xi X=\beta X$.

\section{REFERENCES}

1. H. Cook, Continua which admit only the identity mapping onto non-degenerate subcontinua, Fund. Math. 60 (1967), 241-249. MR 36 \#3315.

2. J. Dugundji, Topology, Allyn and Bacon, Boston, Mass., 1966. MR 33 \#1824.

3. L. Gillman and M. Jerison, Rings of continuous functions, University Series in Higher Math., Van Nostrand, Princeton, N.J., 1960. MR 22 \#6994.

Department of Mathematics, Cleveland State University, Cleveland, Ohio 44115 